\title{
Autoevidencia o precomprensión en el aprendizaje del proyecto arquitectónico. Experiencias anteriores relacionadas con nociones como paisaje, territorio o hábitat
}

\author{
Self-evidence or pre-comprehension in the learning process \\ of the architectonic project. Former experiences related with \\ notions like landscape, territory or habitat
}

Rafael Francesconi Latorre*

Citar este artículo como: Francesconi, L. (2016). Autoevidencia o precomprensión en el aprendizaje del proyecto arquitectónico. Experiencias anteriores, relacionadas con nociones como paisaje, territorio o hábitat. Revista nodo, 11(21), 50-62.

\section{Resumen}

El presente trabajo evaluó el potencial de un concepto de "precomprensión" para aclarar el sentido en el cual nociones como paisaje, territorio o hábitat, ayudarían a anticipar experiencias previas, útiles en el aprendizaje del proyecto arquitectónico y a una sucesión de fuentes filosóficas (Heidegger, Gadamer y Habermas). Con base en estas fuentes caracterizó un concepto de precomprensión, que supone un reconocimiento de la institución arquitectónica y ofrece indicaciones para identificar a posteriori experiencias anteriores pertinentes, para asociar resultados de aprendizaje con experiencias anteriores (a través de un encadenamiento entre experiencias, nociones, conceptos y su aplicación proyectual) y para articular una comparación con estilos de aprendizaje, como explicación alternativa de dichos resultados de aprendizaje.

Palabras clave: Arquitectura, Proyecto, Aprendizaje, Experiencias previas, Paisaje, Gadamer.

\section{Abstract}

This paper evaluates the potential concept of precomprehension to clarify the sense in which notions such as landscape, territory or habitat could help to foresee former experiences useful in the learning process of the architectonic project. For this purpose, sources were used from the pedagogy of architectural project and linked with philosophical ones (Heidegger, Gadamer and Habermas). Based on these sources, a concept of pre-comprehension was characterized, which helped to recognize the existence of an architectural institution and provide clues to an ex post identification of relevant former experiences, which associated results of learning with previous experiences (by linking experiences, notions, concepts and their application on architectural projects) and compared them with learning styles, as an alternative explanation of learning results.

Key words: Architecture, Project, Learning, Previous Experience, Landscape, Gadamer. 


\section{Introducción}

Este artículo trata sobre la utilidad de experiencias anteriores, relacionadas con nociones como paisaje, territorio o hábitat, en el aprendizaje del proyecto arquitectónico. Se desarrolló como parte del proyecto de investigación "El concepto de paisaje en el aprendizaje de la composición arquitectónica" adelantado de manera conjunta por las Universidades Católica de Colombia y Piloto de Colombia, durante 2015. En este contexto, plantea una discusión a la posibilidad de un conocimiento autoestructurante, como el que ha sido sostenido por los investigadores de la primera de estas instituciones de educación superior (Correal \& Verdugo, 2011).

El constructivismo y el social constructivismo, conciben el aprendizaje como un proceso que no parte de cero, sino de afectos, conocimientos y experiencias previas. Con respecto al aprendizaje del proyecto arquitectónico, Jiménez (2006) expone el fundamento pedagógico de su trabajo, para lo cual caracteriza tres "modelos pedagógicos": el conductista, el constructivista y el social-constructivista. Sobre éstos, argumenta que "[l]os modelos pedagógicos han venido desplazándose desde el conductista, centrado en objetivos y desarrollo de destrezas, hacia los otros dos, donde enseñar a pensar -más que enseñar a hacer o instrumentalizar-, implica la liberación en el acto formativo." (Jiménez, 2006, p. 50) En concordancia con la superioridad que atribuye a los modelos constructivista y socialconstructivista, se muestra crítica con respecto a la pervivencia del conductista en la enseñanza del proyecto arquitectónico en Colombia, cuando dice:

El modelo, común en la educación colombiana, desarrolla una enseñanza dirigida y centrada en el docente, quien da las instrucciones y las guías para la solución y construcción del objeto, a un sujeto pasivo que las ejecuta. La evaluación es cuantitativa y se centra en el resultado, es decir, en la valoración del objeto arquitectónico producido, de cuya calidad se infiere la del alumno (Jiménez, 2006, p. 48). ${ }^{1}$

A las limitaciones del modelo conductista, contrapone la concepción como proceso del constructivista que "[a]sume el conocimiento como una construcción propia del sujeto, que se va produciendo día a día como resultado de la interacción a través de diversos contextos en los que se desarrollan sus actividades. Se basa en la construcción de procesos cognitivos y la adquisición de competencias [...]" (Jiménez, 2006, p. 48). De acuerdo con esta interpretación, el modelo conductista asumiría que el aprendizaje parte de cero, mientras que el constructivista siempre encontraría un estado inicial distinto del vacío. Esta manera de concebir el aprendizaje como un proceso que nunca parte de cero, le lleva señalar la necesidad de una pedagogía que reconozca "[...] al sujeto educable [...]" con "[...] sus conocimientos previos e intereses [...]" (Jiménez, 2006, p. 51)². En este sentido, resulta plausible presumir la exis-

1 Esta actitud, recelosa hacia el papel del docente, tiene un contrapunto en investigaciones sobre "cómo, tanto en el pasado como en la actualidad, los artistas educadores han usado su personalidad y experiencia para persuadir a los estudiantes de arte y diseño de que confíen en su juicio" (Owen, 2014, p. 202). De acuerdo, con un trabajo al respecto de Owen (2014) "Parece que el alineamiento del encuadre resulta de un cambio en la interpretación de la orientación individual del estudiante y del líder [profesor], de tal modo que los intereses, valores y creencias de los seguidores [estudiantes] y las actividades, metas e ideología del líder [profesor] (los encuadres) se alineen (p. 207)", esto es particularmente relevante para lo concerniente al papel del profesor, consistente en sacar a los estudiantes de su zona de confort, en persuadirlos para que piensen en nuevas direcciones y esto, se puede argumentar, "es un proceso clave en enseñar a los estudiantes a pensar de manera creativa" (p. 208).

$2 \mathrm{Al}$ modelo social-constructivista se le atribuye un carácter crítico, que se suma al de proceso, reconocido al constructivista: "Se reconoce al sujeto como un ser situado en la historia y la sociedad, y como un ser capaz de razonar, argumentar y comunicarse; intervienen la ética, las proposiciones de cambios pedagógicos basados en las finalidades y la ruptura de arraigos dogmáticos cimentados en "verdades absolutas", dando paso a cuestionamientos, reflexiones y críticas." (Jiménez Correa, 2006, p. 49). 
tencia de una utilidad de experiencias anteriores, relacionadas con nociones como paisaje, territorio o hábitat, en el aprendizaje del proyecto arquitectónico.

Estas presunciones parecen encontrar una corroboración en la práctica. Tal sería el caso expuesto por Uribe (2011), de acuerdo con cuyos argumentos:

[...] la práctica académica de la Escuela de Arquitectura de la Universidad de Talca, durante este último tiempo, ha logrado poner en valor una experiencia previa de habitar y construir, mediante la relectura de un sistema de relaciones complejas. La nueva narrativa arquitectónica asociada al Valle Central de Chile reproduce las sensaciones y la experiencia espacial y cultural de la arquitectura vernácula. La redescripción mantiene la experiencia original de habitar. Redescribir tiene que ver con el reconocer y reconocerse. No es rehacer una tipología o forma arquitectónica, sino que rehacer la esencia en su manera de habitar (Uribe Ortiz, 2011, p. 73).

Este "poner en valor la experiencia de habitar en el Valle Central de Chile" proporcionaría una confirmación de la utilidad de las experiencias anteriores, relacionadas con nociones como paisaje, territorio o hábitat, en el aprendizaje del proyecto arquitectónico.

Sin embargo, los autores mencionados no ofrecen suficientes argumentos que permitan establecer qué tan razonable es atribuir utilidad, en el aprendizaje del proyecto arquitectónico, a experiencias anteriores relacionadas con las nociones indicadas. Por lo anterior, resulta pertinente preguntar: ¿en qué sentido, nociones como territorio, hábitat o paisaje, ayudarían a anticipar experiencias previas, útiles en el aprendizaje del proyecto arquitectónico ${ }^{3}$ Como respuesta a esta pregunta

3 Gregotti (1972), aunque hace un uso metafórico de la expresión "territorio" en el título del libro, lo cual es consistente con su reconocimiento de una autonomía para el proyecto arquitectónico (“[...] creemos en la posibilidad y validez de una temática legítimamente autónoma sobre el proyectar arquitectónico [...] (p. 15), también hace referencia
Imagen 1. Cerro La Virgen, Talca. La obra imita la figura del viñedo en el que se encuentra.

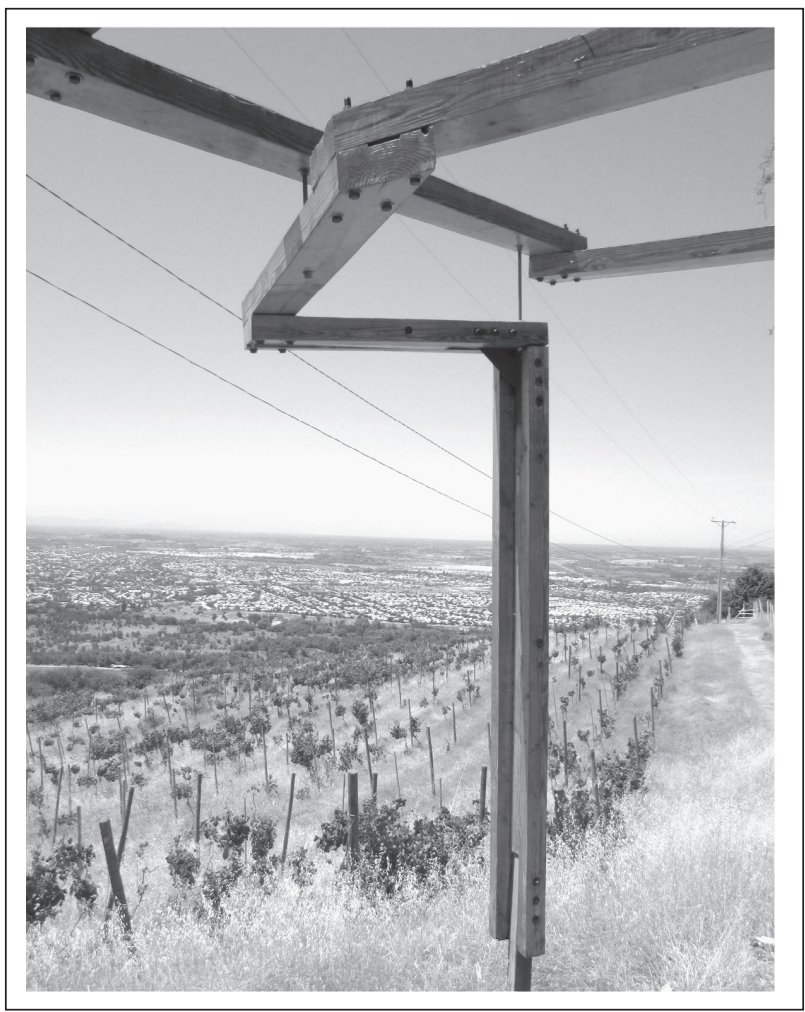

Fuente: fotografía tomada por el autor.

al "[...] problema de la forma arquitectónica del territorio [...]" (p. 95), en consecuencia con su consideración de "[...] nuestro trabajo de arquitectos como trabajo sobre conjuntos ambientales a todas las escalas dimensionales" (p. 69). Bajo esta consideración articula el concepto de arquitectura con conceptos de territorio, hábitat y paisaje. Así como establece una relación entre arquitectura y territorio, plantea un vínculo entre arquitectura y hábitat, cuando dice que "[l]a arquitectura se nos presenta, por tanto, como una respuesta significativa, es decir, poética, al problema del habitar, proyectando y construyendo todo ambiente físico de acuerdo con este objetivo." (pp. 53-54). También plantea una relación entre su concepto de arquitectura con de paisaje en su propuesta de "[...] investigar acerca de la fundamentación de una tecnología formal del paisaje antropogeográfico desde el punto de vista arquitectónico." (p. 69) Esta investigación incluye la pregunta: "¿de qué forma nuestra percepción del paisaje se convierte en percepción estética, es decir, de qué forma se adquiere la conciencia de la cualidad figurativa del paisaje?" (p. 73), para la cual ofrece dos respuestas: "[...] la idea de mito [...]" y "[...] la representación del paisaje [...]" (p. 73). En contraste con una propuesta de articulación como esta, las mencionadas parecen confiadas a algo así como una cierta autoevidencia de nociones como territorio, hábitat y paisaje, así como de las relaciones entre éstas. 
se plantea la hipótesis según la cual un concepto de "precomprensión" contribuye a aclarar el sentido en el cual nociones como territorio, hábitat o paisaje, ayudarían a anticipar experiencias previas, útiles en el aprendizaje del proyecto arquitectónico, $y$, de manera consecuente, (a) suministra indicaciones para la identificación de dichas experiencias anteriores, (b) aporta una base para asociar, de manera razonable, resultados de aprendizaje con experiencias anteriores y (c) ofrece la posibilidad de establecer comparaciones con explicaciones alternativas.

En consecuencia con dicha hipótesis, este trabajo aboca el objetivo de evaluar el potencial de un concepto de "precomprensión" para aclarar el sentido en el cual nociones como paisaje, territorio o hábitat, ayudarían a anticipar experiencias previas, útiles en el aprendizaje del proyecto arquitectónico. Para alcanzar dicho objetivo se requiere caracterizar un concepto de "precomprensión" y evaluar sus capacidades para:

a. Identificar experiencias anteriores, relacionadas con nociones como paisaje, territorio o hábitat y establecer condiciones para su registro.

b. Aportar una base para asociar, de manera razonable, resultados de aprendizaje con experiencias anteriores.

c. Sustentar comparaciones con explicaciones alternativas.

La evaluación indicada se llevó a cabo mediante la interpretación de fuentes secundarias, seleccionadas con criterios de pertinencia: Se empleó fuentes tomadas de la pedagogía del proyecto arquitectónico, para establecer los antecedentes y delimitar el problema (lo cual fue adelantado en esta introducción); se acudió a una sucesión de fuentes filosóficas para caracterizar un concepto de precomprensión (lo que se presenta en el apartado Metodología, por corresponder a la adaptación, al caso en consideración, de un aparato conceptual desarrollado en un ámbito más amplio, con lo que se logró el componente del objetivo, correspondiente a dicha caractariza- ción) y a fuentes de la disciplina de la arquitectura en general y de su pedagogía, para identificar el contexto y los supuestos que éste implicaba para su aplicación al aprendizaje del proyecto arquitectónico (recogidos en la sección resultados, como logro de los objetivos específicos a los que se refieren los literales (a), (b) y (c), anteriores $)^{4}$.

\section{Metodología}

El concepto de pre-comprensión se introduce a continuación, contrastándolo con una aproximación que presupone la existencia de hechos evidentes por sí mismos, que son captados en el aprendizaje de la ciencia.

La consideración con respecto a "[e]n qué medida lo que un estudiante dado aprende en clase depende de sus habilidades innatas o de la preparación previa, o también de la compatibilidad de su estilo de aprendizaje con el estilo de enseñanza de su profesor" guio la reflexión de Felder y Silverman (1988, p. 674). De manera consecuente con su origen en el campo de la educación en ingeniería, este artículo, reconocido como un trabajo pionero en el estudio de los estilos de aprendizaje, concibe el aprendizaje formal de una disciplina como "[...] un proceso en dos pasos que incluye la recepción y el procesamiento de información” (Ídem.). Con respecto a este símil sus autores definieron " $[\mathrm{u}] \mathrm{n}$ modelo de estilos de aprendizaje [que] clasifica a los estudiantes de acuerdo a dónde encajan en una serie de escalas relativas a las formas como reciben y procesan la información". Con base en fuentes secundarias, el artículo documentó dos desajustes entre estilos de aprendizaje y estilos de enseñanza: predominio de profesores "intuitivos" en contraste con una mayoría de estudiantes "sensitivos" y preponderancia de estudiantes "visuales" enfrentados a contenidos académicos verbales (p. 676).

4 Cabe anotar que el recurso a fuentes filosóficas, tiene un interés instrumental, que no implica que la teoría de la arquitectura se subordine a una ciencia particular, como, por ejemplo, la antropología en según se indica más adelante, en la nota 9 (Egenter, 2006, p. 30). 
Mediante encuestas informales propias, identificó una más, entre aprendizaje inductivo y enseñanza deductiva. Entre sus recomendaciones incluyó la de "[s]eguir el método científico al presentar el material teórico" (p. 680). En el prefacio escrito en 2002, Felder anota que el artículo "empezó a ser citado en la literatura sobre educación en ingeniería y luego en aquella sobre educación en ciencias en general", lo que indicaría su grado de adecuación al contexto científico.

A este tipo de comprensión del proceso del aprendizaje a partir de una metáfora computacional, adecuada para el de la ingeniería y las ciencias, se puede proponer una alternativa, desde una comprensión hermenéutica del aprendizaje del proyecto arquitectónico. Al respecto, cabe anotar que Verdad y método (1996/1975; 1994/1986) de Hans-Georg Gadamer, contrapone la hermenéutica a la ciencia del método ("pensamiento analítico") y que su punto de partida es la manera en la que el arte conoce. Al hacer referencia a la "Significación de la tradición humanística para las ciencias del espíritu”, este autor aboga por la especificidad de este tipo de ciencias en los siguientes términos:

$\mathrm{Ni}$ el moderno concepto de la ciencia ni el concepto de método que le es propio pueden bastar. Lo que convierte en ciencias a las del espíritu se comprende mejor desde la tradición del concepto de formación que desde la idea de método de la ciencia moderna. En este punto nos vemos remitidos a la tradición humanista, que adquiere un nuevo significado en su calidad de resistencia ante la pretensiones de la ciencia moderna (Gadamer, 1996/1975, p. 47).

Por su parte, en relación con la existencia de una verdad propia del arte, que escapa al método científico, anota en la introducción al primer tomo de la obra en referencia:

La investigación científica que lleva a cabo la llamada ciencia del arte sabe desde el principio que no le es dado ni sustituir ni pasar por alto la experiencia del arte. El que en la obra de arte se experimente una verdad que no se alcanza por otros caminos es lo que hace el significado filosófico del arte, que se afirma frente a todo razonamiento. Junto a la experiencia de la filosofía, la del arte representa el más claro imperativo de que la conciencia reconozca sus límites (Gadamer, 1996/1975, p. 24).

Gadamer encuentra un antecedente para sus planteamientos sobre la precomprensión en la "preesturctura de la comprensión" a la que hace referencia Heidegger en Ser y tiempo (2006 [1927]). En relación con este antecedente, señala una diferencia con respecto a su propio trabajo cuando dice que Heidegger restringe su consideración a una cuestión ontológica, con respecto a la cual él plantea una ampliación para abarcar la "historicidad de la comprensión"; en sus términos:

Heidegger sólo entra en la problemática de la hermenéutica y críticas históricas con el fin de desarrollar a partir de ellas desde el punto de vista ontológico la preestructura de la comprensión [M. Heidegger, Sein und Zeit, pp. 312 y ss.]. Nosotros, por el contrario, perseguiremos la cuestión de cómo, una vez liberada de las inhibiciones ontológicas del concepto científico de la verdad, la hermenéutica puede hacer justicia a la historicidad de la comprensión (Gadamer, 1996/1975, p. 331).

Aunque Gadamer remite en este pasaje al parágrafo 63 [La situación hermenéutica alcanzada para una interpretación del sentido del ser del cuidado y el carácter metodológico de la analítica existencial en general] incluye la siguiente cita del parágrafo 32 [Comprender e interpretación] de Ser y tiempo (2006 [1927]), en la que se hace referencia al problema de la circularidad:

El círculo no debe ser degradado a círculo vicioso, ni siquiera a uno permisible. En él yace una posibilidad positiva del conocimiento más originario, que por supuesto sólo se comprende realmente cuando la interpretación ha comprendido que su tarea primera, última y constante consiste en no dejarse imponer nunca por ocurrencias propias o por conceptos populares ni la posición ni la previsión ni la 
anticipación, sino en asegurar la elaboración del tema científico desde la cosa misma [M. Heidegger, Sein und Zeit, p. 153] (Gadamer, 1996/1975, p. 332). ${ }^{5}$

En consecuencia, con el interés ontológico de la obra de Heidegger citada, el problema de la circularidad se presenta asociado a la búsqueda de un "conocimiento más originario", en virtud del cual se justifica otra forma de "cientificidad" no menos rigurosa, que no sea ni arbitraria (impuesta por "simples ocurrencias") ni convencional (debida a "opiniones populares") sino que se atenga a "las cosas mismas". El mencionado conocimiento más originario puede reivindicar sus derechos frente a otros tipos de conocimiento como el matemático y sus criterios de rigor:

Dado que, en virtud de su sentido existencial el comprender es el poder-ser del Dasein mismo, los supuestos ontológicos del conocimiento histórico trascienden fundamentalmente la idea del rigor de las ciencias más exactas. La matemática no es más rigurosa que la historia, sino tan sólo más estrecha en cuanto al ámbito de los fundamentos existenciales relevantes para ella (Heidegger, 2006 [1927], pp. 176-177).

En la obra de Heidegger que se comenta, no sólo se señala una distancia con respecto al conocimiento matemático sino en relación con la comprensión común, en particular con respecto a una pretendida autoevidencia de los hechos. Al respecto, el parágrafo 63 señala:

5 La traducción de este mismo pasaje para la edición de Ser y tiempo, a la que se alude más adelante reza: "No se lo debe rebajar [al círculo del comprender] a la condición de un circulus vitiosus, ni siquiera a la condición de un círculo vicioso tolerado. En él se encierra una positiva posibilidad del conocimiento más original, posibilidad que, sin embargo, sólo será asumida de manera auténtica cuando la interpretación haya comprendido que su primera, constante y última tarea consiste en no dejar que el haber previo, la manera previa de ver y la manera de entender previa les sean dados por simples ocurrencias y opiniones populares, sino en asegurarse el carácter científico del tema mediante la elaboración de esa estructura de prioridad a partir de las cosas mismas" (Heidegger, 2006 [1927], p. 176).
Lo característico de la comprensión común consiste en que ella cree experimentar tan sólo entes "de hecho", para poder así sustraerse a una comprensión del ser. No comprende que los entes sólo pueden ser experimentados "de hecho" si el ser ya ha sido comprendido, aunque sea sin conceptos. La comprensión común comprende mal el comprender. Y por eso tiene que considerar necesariamente, como "violento" aquello que supera el alcance de su comprensión e incluso la tendencia misma a superarla (Heidegger, 2006 [1927], p. 334).

Una consideración más amplia del pasaje citado por Gadamer permite reconocer algunas pautas, que no dependerían de su discusión ontológica y que corresponderían a una mejor compresión de lo que significa la comprensión misma.

Sin embargo ver en este círculo un circulus vitiosus y buscar cómo evitarlo, o por lo menos, "sentirlo" como imperfección inevitable, significa mal comprender radicalmente el comprender. No se trata de adecuar el comprender y la interpretación a un determinado ideal de conocimiento, que no es sino una variedad del comprender que se ha orientado hacia la legitima empresa del aprehender lo que está-ahí en su esencial incomprensibilidad. Por el contrario, el cumplimiento de las condiciones fundamentales de toda interpretación exige no desconocer de partida las esenciales condiciones de su realización. Lo decisivo no es salir del círculo, sino entrar en él en forma correcta. Este círculo del comprender no es un circuito en el que gire un género cualquiera de conocimiento, sino que es la expresión de la estructura existencial de prioridad del Dasein mismo (Heidegger, 2006 [1927], p. 176).

Este llamado a entrar de manera correcta en el círculo de la comprensión, sería un aporte a la cuestión de la precomprensión. Otros aportes habrían sido identificados por Gadamer en el reconocimiento "conceptos previos" como punto de partida para la interpretación y su convalidación, como criterio de objetividad. El reconocimiento del papel que desempeñan los conceptos previos en el proceso de comprensión e interpretación lo atribuye Gadamer a Heidegger, cuando dice: 
[...] la interpretación empieza siempre con conceptos previos que tendrían que ser sustituidos progresivamente por otros más adecuados. Y es todo este constante reproyectar, en el cual consiste el movimiento de sentido del comprender e interpretar lo que constituye el proceso que describe Heidegger (Gadamer, 1996/1975, p. 333). ${ }^{6}$

A continuación, propone un criterio que da sentido a la progresiva adecuación de los conceptos que han de sustituir aquéllos previos, la confirmación "en las cosas": "El que intenta comprender está expuesto a los errores de opiniones previas que no se comprueban en las cosas mismas. Elaborar los proyectos correctos y adecuados de las cosas, que como proyectos son anticipaciones que deben confirmarse "en la cosas", tal es la tarea constante de la comprensión." (Gadamer, 1996/1975, p. 333). Este criterio de corrección progresiva restringe la arbitrariedad de la interpretación, y hace posible la distinción de opiniones previas no arbitrarias, como argumenta a continuación de lo anterior, el pasaje citado:

Aquí no hay otra objetividad que la convalidación que obtienen las opiniones previas a lo largo de su elaboración. Pues ¿qué otra cosa es la arbitrariedad de las opiniones previas inadecuadas sino que en el proceso de su aplicación acaban aniquilándose? La comprensión sólo alcanza sus verdaderas posibilidades cuando las opiniones previas con las que se inicia no son arbitrarias (Gadamer, 1996/1975, p. 333).

Al respecto, cabe preguntar si se puede saber de antemano cuáles conceptos previos no son arbitrarios. La respuesta que ofrecería el mismo texto sería negativa, puesto que el criterio que ofrece para identificar las "opiniones previas inadecuadas" es su aniquilación en el proceso de comprensión e interpretación. No obstante este criterio de valida-

6 El texto de Gadamer hace referencia a "conceptos previos" y "opiniones previas", no a "experiencias previas", al respecto cabe preguntar si tales conceptos y opiniones serían las experiencias mediadas por su comunicabilidad, aunque habría que reconocer que podrían existir "conceptos previos" y "opiniones previas" no vinculadas a experiencias, sino recibidas por medio del lenguaje. ción en el mismo proceso, el texto que se comenta incluye la recomendación de revisar la legitimidad, origen y validez de las opiniones previas: "Por eso es importante que el intérprete no se dirija hacia los textos directamente desde las opiniones previas que lo subyacen, sino que examine tales opiniones en cuanto a su legitimación, esto es, en cuanto a su origen y validez" (Gadamer, 1996/1975, pp. 333-334).

Además de reconocer un antecedente en la mencionada preestructura de la comprensión, que encontró en Ser y tiempo (Heidegger, 2006 [1927]), Gadamer identifica un aporte en el proceso de aplicación de la hermenéutica en contextos diferentes del de la reflexión ontológica, en particular en el campo de las ciencias sociales, en Habermas (2001 [1988 ]). Al respecto Gadamer dice:

Debo a la lúcida labor de Habermas el haber percibido con claridad la aportación hermenéutica dentro de las ciencias sociales, especialmente cuando compara la precomprensión de la teoría positivista de la ciencia, pero también la de una fenomenología apriorista y de una lingüística general, con la dimensión hermenéutica" (Gadamer, 1994/1986, p. 239).

En el texto al que hace referencia Gadamer, apunta Habermas hacia las dificultades de la comprensión de "plexos simbólicos" legados por la tradición.

La comprensión del sentido se torna metodológicamente problemática cuando se trata de apropiación de contenidos semánticos legados por la tradición: el «sentido» que ha de explicitarse tiene entonces el status de un hecho, de algo empíricamente ahí. La comprensión de los plexos simbólicos que nosotros mismos generamos es aproblemática. Así, los enunciados formalizados, ya sean proposiciones matemáticas o teorías en sentido estricto, no nos ponen, como los textos o documentos recibidos, ante las tareas típicas de la interpretación hermenéutica. $Y$ es que a los lenguajes formalizados pertenecen las reglas metalingüísticas de constitución con cuya ayuda reconstruimos los enunciados dados, esto es, los generamos de nuevo. El pensamiento 
analítico puede ser contrapuesto con toda razón a la discusión hermenéutica (Habermas, 2001/1988, p. 173).

Esta observación pondría en cuestión una eventual atribución de un carácter de autoevidencia a nociones como paisaje, territorio o hábitat, y permite entender que aunque su sentido pueda presentarse con el "status de un hecho, de algo empíricamente ahí", ponen a quienes han de comprender su sentido "ante las tareas típicas de la interpretación hermenéutica”. Por su parte, la observación final con respecto a la contraposición entre "pensamiento analítico" y "discusión hermenéutica" daría la razón a haber apostado por la diferencia entre éstas, en lugar de haber tomado opciones que suponen una mayor afinidad entre filosofía, ciencias sociales y actividad artística, como el discurso de la ciencias de la complejidad.

De acuerdo con lo anterior, se puede caracterizar un concepto de precomprensión a partir de:

a. El reconocimiento de una circularidad en el proceso de comprensión, que no se puede evitar;

b. Por lo cual, dicho proceso ha de aceptar conceptos previos como punto de partida (como una manera apropiada de ingresar en el círculo de la comprensión);

Imagen 2. Fotografía sin título, estructuras hechas por Diego Vergara en 2011 (Talca).

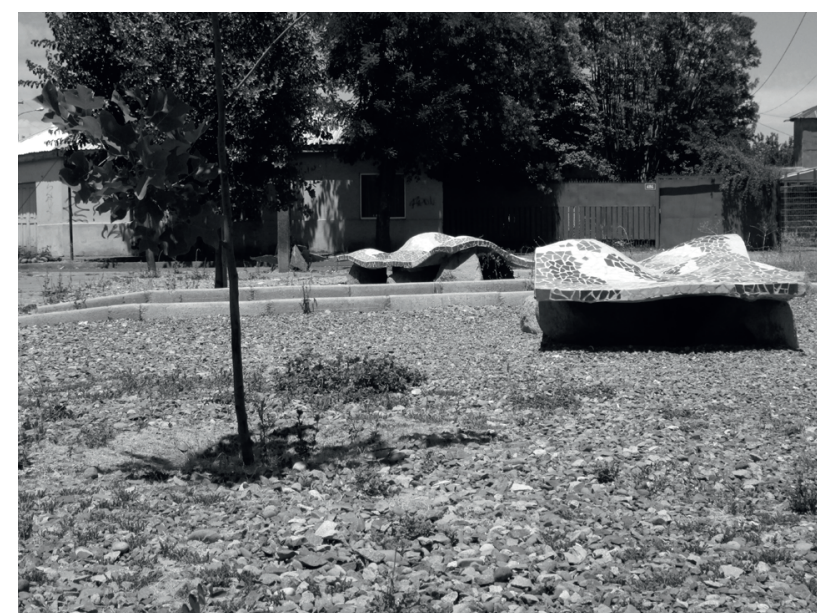

Fuente: fotografía tomada por el autor. c. A la vez que se propone distinguir opiniones previas "que se comprueban en las cosas mismas" de aquellas inadecuadas;

d. Para lo que tendría, como criterio de objetividad, la convalidación de las opiniones previas a lo largo de su elaboración;

e. Así como el examen de tales opiniones en cuanto a su "legitimación, esto es, en cuanto a su origen y validez".

Por otra parte, la caracterización del concepto de precomprensión efectuada invita a aceptar que los discursos de la disciplina de la arquitectura, desarrollados a partir de nociones como paisaje, territorio o hábitat, requieren que su aprendizaje los aborde como un proceso de interpretación de un sentido legado por la tradición.

\section{Resultados}

Con esta caracterización, se alcanza el primero de los objetivos específicos planteados (caracterizar un concepto de "precomprensión"). Resta, entre otros objetivos específicos pendientes, evaluar las capacidades de ésta, para guiar la identificación experiencias anteriores, relacionadas con nociones como paisaje, territorio o hábitat. La referencia a la circularidad de la comprensión anticipó una dificultad debida a la imposibilidad de para identificar ex ante cómo sería una experiencia previa que conduzca al aprendizaje de conceptos como los de paisaje, territorio o hábitat, acuñados por la disciplina de la arquitectura, siendo forzoso llevar a cabo tal identificación a posteriori. Una identificación de este tipo cae bajo la sospecha de la explicación post factum, es decir, de que siempre será posible acomodar una explicación a los hechos ya acaecidos. Para limitar, hasta donde sea posible, esta sombra de sospecha, resultan útiles las notas de la caracterización efectuada con respecto a (a) la distinción de "opiniones previas" que se "comprueban en las cosas mismas", de las opiniones inadecuadas; (b) la convalidación de dichas opiniones previas a lo largo de su elaboración, como criterio de objetividad, y (c) el examen 
de tales opiniones "en cuanto a su legitimación [...] origen y validez". Ahora bien, la aplicación estás notas implica despejar, en contexto del aprendizaje del proyecto arquitectónico, el sentido de "comprobación en las cosas mismas", "convalidación a lo largo de su elaboración" y "examen en cuanto a legitimación, origen y validez". La referencia a dicha "cosa misma" se encontraría matizada por la misma caracterización efectuada, como un "contenido [...] legado por la tradición", que implicaría que la comprobación requerida se lleve a cabo en el contexto del respectivo contenido legado por la tradición. Por su parte, una convalidación de las opiniones previas a lo largo de la elaboración sugiere una aproximación entre el criterio de objetividad propuesto, y un criterio de coherencia (algo así como una teoría coherentista de la verdad). La aplicación del criterio de coherencia implica la subordinación de la opinión previa al contenido recibido de la tradición? ${ }^{7}$ Estas

7 La relación con el legado de la tradición ha sido objeto de la teoría de la arquitectura, como en el trabajo de Jorge Francisco Liernur: "La reivindicación de la arquitectura en el sentido institucional tradicional, o more Vitruviana como la ha caracterizado Kurt Forster en el texto citado más arriba ["Thoughts on the metamorphoses of architecture", Log, No. 3 (Fall 2004), pp. 19-27], obedece a la necesidad de hacer ingresar nuestra concepción de esta tarea ese rol temporal -nave del tiempo, la llamaría Mishima- que le corresponde asumir a la disciplina. La vapuleada institución arquitectónica supone, entre otras cosas la posibilidad de recibir esa dote de la que habla Benjamin ["Hay un acuerdo secreto entre las generaciones pasadas y la nuestra. Nosotros hemos sido esperados en la tierra. A nosotros como a cada generación que nos ha precedido, se nos ha dado en dote una débil fuerza mesiánica, sobre la cual el pasado tiene un derecho." Tesis de filosofía de la historia, Madrid, 1973 (1 ed. Frankfurt, 1969)], introducirle las marcas de nuestra propia existencia y volver a legarla a futuras generaciones. Esa transmisión no es posible sin códigos comunes, aunque como el lenguaje éstos puedan (y deban) ir modificándose siempre." (Liernur, 2010, p. 229) La referencia "la institución arquitectónica" sugiere que la comprensión que requiere la aplicación de experiencias previas, relacionadas con nociones como paisaje, territorio o hábitat, es análoga a la que requería el aprendizaje de la ciencia de la economía a partir de las experiencia de haber vivido en medio de relaciones sociales de producción, en el sentido de que la ciencia económica referencias a contenidos legados por una tradición, a su vez, orientan el mencionado examen de legitimidad, en el sentido de indicar que la validez de las nociones previas dependerá de su coherencia con los respectivos contenidos recibidos de la tradición y de su aporte a un tránsito desde estas nociones, hacia conceptos reconocidos por la respectiva tradición.

Dentro de los objetivos específicos también se incluyó el de aportar una base para asociar resultados de aprendizaje con experiencias anteriores. En relación con este objetivo, el concepto de precomprensión caracterizado implica, para el aprendizaje del proyecto arquitectónico, el tránsito a través de una serie de momentos: el primero de la experiencia a la noción; el segundo, de ésta al concepto; el tercero, del concepto a su aplicación proyectual. De esta manera, la aclaración del sentido en el cual nociones como territorio, hábitat o paisaje, ayudarían a anticipar experiencias previas, útiles en el aprendizaje del proyecto arquitectónico, requería considerar cada uno de dichos momentos de tránsito, es decir, distinguiría entre dos niveles aprendizaje y de utilidad de las experiencias anteriores: el primero, correspondiente al paso de la noción al concepto y el segundo, al que se da entre el concepto y su aplicación ${ }^{8}$.

puede reconstruir las relaciones sociales de producción correspondientes a contextos en los que en estricto sentido no existía una ciencia de la economía, así, conceptos tomados del legado de la institución arquitectónica pueden ser aplicados en contextos históricos o contemporáneos, en los que no se reconoce la institución arquitectura, dando lugar a una interpretación antropológica de la arquitectura. De esta manera, la utilidad de experiencias anteriores, relacionadas con nociones como paisaje, territorio o hábitat, en el aprendizaje del proyecto arquitectónico, dependería de un tránsito desde una precomprensión antropológica de la arquitectura hacia una comprensión como la legada por la institución arquitectura.

8 Una de fuente a la que recurre la literatura sobre la arquitectura del paisaje menciona una distinción análoga a la que estaría implicada en al paso de la noción al concepto: "En este caso particular [tener presente que la idea de "tener una comprensión de" no requiere una referencia a una capacidad de pensamiento propiamente dicha] significa, desde luego, 
Con respecto al objetivo específico, consistente en permitir comparaciones con explicaciones alternativas, cabe anotar que el concepto de precomprensión caracterizado, se aproximó desde el qué del proceso de aprendizaje, lo que ofrece una oportunidad de complementación desde el quién. Una aproximación desde el quién del aprendizaje se encontraría en las investigaciones sobre estilos cognitivos, de las cuales hace parte el trabajo de Felder y Silverman (1988), antes mencionado.

El concepto de precomprensión caracterizado está en capacidad de guiar la identificación $a$ posteriori de experiencias anteriores, relacionadas con nociones como paisaje, territorio o hábitat, que hayan resultado útiles en el aprendizaje del proyecto arquitectónico. Las experiencia así identificadas se comprobarían en relación con el contexto representado por alguna tradición disciplinar, mediante la verificación de su coherencia con dicha tradición; mientras que su utilidad para el aprendizaje se establecería con base en su contribución a un tránsito, desde estas nociones, hacia conceptos reconocidos por la tradición respectiva. En relación con la provisión de una base para la asociación entre resultados de aprendizaje y experiencias anteriores, el concepto de precomprensión en referencia aporta una distinción de momentos en el proceso de aprendizaje del proyecto arquitectónico, con respecto a los cuales se establecería un grado de asociación. $\mathrm{Y}$, en relación con la capacidad para articular comparaciones con explicaciones alternativas, el concepto de precomprensión caracterizado resulta complementario con investigaciones sobre estilo cognitivo. En el sentido así sintetizado, se alcanzó el objetivo general evaluar el potencial del concepto de precomprensión caracterizado,

que la afirmación de que cierta criatura tiene una comprensión del espacio no puede ser tomada como si incluyera la afirmación adicional de que la criatura en referencia tiene un concepto o una idea de espacio [...]. Debemos distinguir, por lo tanto, entre una comprensión del espacio que involucra una comprensión del concepto de espacio y la comprensión que consiste en la posesión simplemente de ciertas capacidades o disposiciones de comportamiento" (Malpas, 1999, p. 46). para aclarar el sentido en el cual nociones como territorio, hábitat o paisaje, ayudarían a anticipar experiencias previas, útiles en el aprendizaje del proyecto arquitectónico.

\section{Discusión}

Como se indica a continuación, desde tres puntos de vista se podría discutir las implicaciones del concepto de precomprensión aquí caracterizado: el de cierta elusión de la fatiga del concepto, el de la antropología arquitectónica y el del pensamiento de diseño.

El tránsito indicado entre experiencia, noción, concepto y aplicación, atribuye al proceso de aprendizaje del proyecto arquitectónico un momento de "conceptualización", como uno de sus constitutivos ineludibles. En el contexto latinoamericano, este supuesto sería objeto de discusión, como en el mencionado caso de Talca, que reporta entre sus orígenes la elusión de las dificultades relacionadas con la comprensión y aplicación del concepto arquitectónico de espacio ${ }^{9}$. Desde un punto de

9 "Había una capacidad de abstracción muy reducida y Román la enfocaba a los temas más generales, para enseñarles a ver, a orientar la mirada hacia los valores de la arquitectura, a dilucidar de qué hablamos cuando hablamos de espacio. [...] Pero resulta que el espacio, como concepto, quedaba muy lejos. Juan Román recuerda que "en la escuela no se hablaba de espacio, sino de "espaaaacio", como una cosa de origen divino, y mientras estudiaba - recuerda- nunca lo vi, lo viene a ver como a los 44 años. Quizá al ejercer de arquitecto estuve estafando gente ya que nunca había visto el "espaaaacio"." [...] Un paso clave en todo el proceso de gestación de este proyecto académico fue la decisión de abordar la arquitectura desde la experiencia de lo material y ya no desde la percepción del espacio." (Adriá et al., 2013, p. 19). Esta elusión de las dificultades conceptuales que plantea la disciplina, parece consistente la escasa valoración de la autonomía disciplinar en la región. Por ejemplo, un ejercicio regional para establecer las competencias que deben demostrar los egresados de los programas de pregrado en Arquitectura, entre 55 de éstas, identificadas en una primera fase desarrollada entre 2004 y 2007, relegó a los últimos lugares de la ponderación efectuada en la segunda (que tuvo lugar entre 2011 y 2013) aquella correspondiente al "Conocimiento, sensibilidad y compromiso hacia los problemas del actual debate arquitectónico local y global" (Vélez et al., 2013, p. 78). En un contexto análogo, restringido al caso colombiano, 
Imagen 3. Cierre perimetral multicancha, hecha por Dafne Aristía en 2007 (Talca).

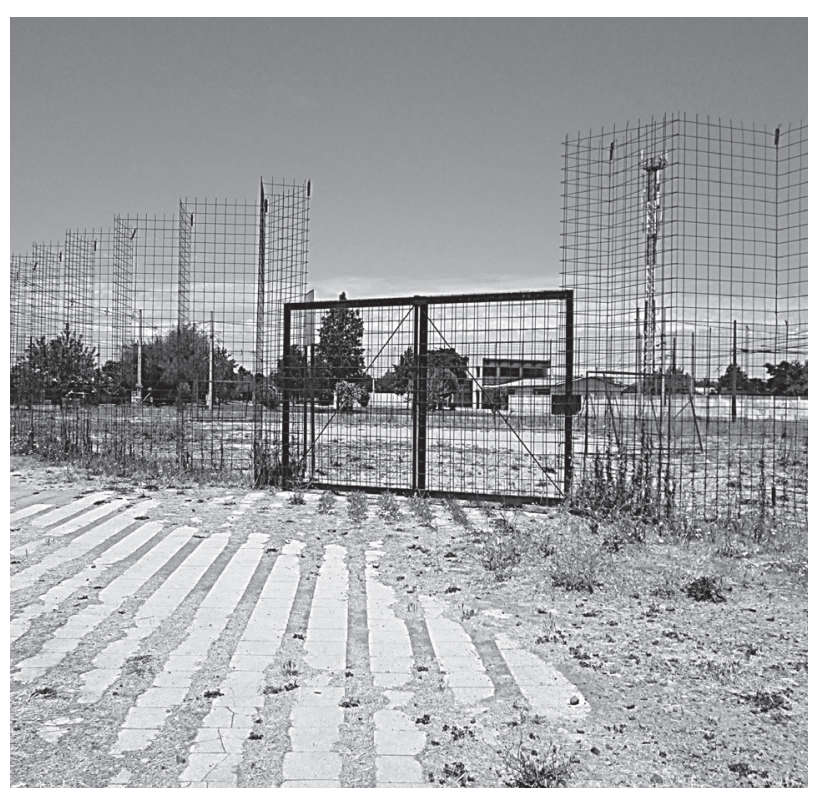

Fuente: fotografía del autor.

vista como este, se rechazaría que sea necesario un tránsito entre la experiencia y la aplicación proyectual de sus contenidos, a través de la noción y el concepto. Esta anotación sugiere la conveniencia de plantear experimentos que exploren un vínculo directo entre experiencia y aplicación.

En relación con los supuestos del concepto de precomprensión caracterizado, cabe anotar que reconoce una base antropológica para la arquitectura (en el tránsito entre experiencia y noción), pero también la existencia de la institución arquitectónica (cuyo umbral se cruza en el paso entre la noción y el concepto), es decir, rehúsa la reducción de la teoría de la arquitectura a un departamento de la antropología (que sería como negar la autonomía de la literatura, para convertirla en un departamento de la lingüística), como se ha pretendido desde la antropología arquitectónica

se llagó incluso a la disolución de la autonomía disciplinar, mediante una transformación de la competencia mencionada en: "Emprender el trabajo autónomo y colaborativo en ambientes interdisciplinarios, para fortalecer el interés por los temas del debate social y arquitectónico" (Grupo de expertos de la Asociación Colombiana de Facultades de Arquitectura-ACFA, 2011, p. 7). $\left(\right.$ Egenter, 2006) ${ }^{10}$. Para esta antropología arquitectónica, resultaría inaceptable la reivindicación de la autonomía de la "institución arquitectura", la que obligaría que el aprendizaje del proyecto arquitectónico implicara un tránsito entre la noción (explicable en términos antropológicos) y el concepto legado por la tradición de la disciplina (no generalizable en desde un punto de vista antropológico).

Por otra parte, desde el campo correspondiente al pensamiento de diseño se ha argumentado la conveniencia de empezar haciendo tabula rasa. Se ha considerado, por ejemplo que "[...] se puede argumentar que es pedagógicamente apropiado empezar [...] persuadiendo los estudiantes de que "olviden todo lo que han aprendido sobre arte y empiecen desde el principio". (Owen, 2014, p. 210). Desde esta aproximación, se encontraría que la precomprensión caracterizada confiere demasiada importancia a la tradición en el proceso de aprendizaje. Una aproximación como esta representa lo contrario a la valoración de las experiencias anteriores y previene contra una actitud dogmática, que pretenda reducir el aprendizaje del proyecto arquitectónico a una elaboración de nociones, en conceptos legados por alguna tradición, con destino a su aplicación proyectual.

También en prevención de un dogmatismo, cabe remitir un eventual juicio concluyente sobre la utilidad de experiencias anteriores, en el aprendi-

10 Egenter (2006) sostiene que "[...] el horizonte teórico determinado por la historia convencional del arte debe ser expandido" (p. 16) para admitir "[...] una investigación científica y razonada"; por lo que, propone redefinir el término arquitectura para que incluya "todo lo que los seres humanos y sus parientes biológicos construyen y construyeron". Es decir, propone "un concepto de arquitectura definido antropológicamente", cuyo criterio de demarcación es la existencia de "[...] comportamientos constructivos cotidianos (recurrentes) que produzcan alteraciones permanentes de las condiciones naturales del ambiente" (p. 17). Egenter insiste en que "la teoría de la arquitectura es cuestión de antropología" y que "[l]a antropología ha de aclarar la complejidad del dominio arquitectónico en lo que se refiere a la condición humana" (p. 30). 
zaje del proyecto arquitectónico, a los resultados experimentales.

En síntesis, el concepto de precomprensión caracterizado reivindica la necesidad de un tránsito a través de la noción y el concepto, ante la pretensión de un paso directo de la experiencia (del territorio, el paisaje o el hábitat, incluso de una arquitectura vernácula) a la proyección arquitectónica. Así mismo opone la autonomía de la institución arquitectura, tanto frente las pretensiones ("imperialistas") de la antropología arquitectónica, como ante la tabula rasa de algunos sectores del pensamiento de diseño. La discusión entre estos puntos de vista se beneficiaría con experimentación.

\section{Conclusiones}

Se ha caracterizado un concepto de precomprensión a partir de:

a. El reconocimiento de una circularidad en el proceso de comprensión;

b. una necesidad de nociones previas, como punto de partida, $y$

c. una comprobación de la validez de éstos en el contexto del legado de una tradición reconocida por la disciplina, de acuerdo con un criterio de coherencia.

Como ya se puntualizó:

a. La utilidad, para el aprendizaje del proyecto arquitectónico, de las experiencias identificadas con la guía del concepto de precompresión caracterizado, se establecería con base en su contribución a un tránsito, desde nociones como paisaje, hábitat y territorio, hacia conceptos reconocidos por la tradición respectiva.

b. El concepto de precomprensión caracterizado aporta una distinción de momentos en el proceso de aprendizaje del proyecto arquitectónico (en particular, uno en el tránsito entre experiencia y noción, que se mantiene en el ámbito de una "antropología arquitectónica", y otro en el paso entre la noción y el concepto, en el cual se cruza el umbral de la "institución arquitectónica"), con respecto a los cuales se establecería un grado de asociación entre resultados de aprendizaje y experiencias anteriores, de esta manera, proporciona una base para tal asociación.

c. Este concepto de precomprensión resulta complementario con investigaciones sobre estilo cognitivo, y, en esa medida, está en capacidad para articular comparaciones con esta explicación alternativa.

En contraste con un contexto regional (latinoamericano), en el que, al parecer, se desconfía de la utilidad de la elaboración conceptual para el proyecto arquitectónico, y otro más amplio, representado por el "pensamiento de diseño", confiado a la aventura de la innovación, el concepto de precomprensión caracterizado sitúa el aprendizaje de la actividad proyectual en el contexto de la institución arquitectónica, para la cual nociones como las de paisaje, territorio o hábitat, en lugar de gozar de una privilegiada autoevidencia, constituyen una materia prima suministrada, tal vez, por una antropología arquitectónica.

\section{Referencias bibliográficas}

Adriá, M., Cuadra, M., Griborio, A., Mellado, J.P., Román, J., \& Uribe, J.L. (2013). Talca. Cuestión de educación (Primera edición ed.). (J. L. Uribe Ortiz, Ed.) México D. F., México: Arquine S. A. de C.V.

Correal Pachón, G., \& Verdugo Reyes, H. (2011). “Sobre modelos pedagógicos y el aprendizaje del proyecto arquitectónico". Revista de Arquitectura, 13, pp. 80-89.

Egenter, N. (2006). "Anthropology of Habitat and Architecture". Journal of the Development and Research Organisation for Nature, Arts and Heritage, Dronah, Gurgaon, India, pp. 16-31.

Felder, R.M., \& Silverman, L.K. (1988). Learning and teaching styles in engineering education. Engr. Education, 78 (7), pp. 674-681.

Gadamer, H.-G. (1994/1986). Verdad y método II (segunda edición, primera 1992 ed.). (M. Olasagasti, Trad.) Salamanca, España: Ediciones Sígueme S.A.

Gadamer, H.-G. (1996/1975). Verdad y método. I. Fundamentos de una hermenéutica filosófica (Sexta 
edición, primera 1977 ed.). (A. Agud Aparicio, \& R. de Agapito, Trads.) Salamanca, España: Ediciones Sígueme, S.A.

Grupo de expertos de la Asociación Colombiana de Facultades de Arquitectura -ACFA. (2011). "Marco conceptual de la propuesta para la resolución de condiciones especificas que modifique la Resolución 2770 de 2003. Documento Preliminar". Asociación Colombiana de Facultades de Arquitectura -ACFA. Bogotá, D.C.: Asociación Colombiana de Facultades de Arquitectura -ACFA.

Habermas, J. (2001 [1988 ]). III. Sobre la problemática de la comprensión del sentido en las ciencias empíricoanalíticas de la acción. En J. Habermas, La lógica de las ciencias sociales (M. J. Redondo, Trad., pp. 173-256). Madrid: Editorial Tecnos (Grupo Anaya, S.A.).

Heidegger, M. (2006 [1927]). Ser y tiempo. (J.E. Rivera C., Trad.) Madrid, España: Editorial Trotta.

Jiménez, S. (2006). El proyecto arquitectónico aprender investigando (Primera ed.). Cali, Colombia: Universidad de San Buenaventura Cali- Facultad de Arquitectura, Arte y Diseño.

Liernur, F. (2010). "Acerca de la delicadeza: consideraciones sobre la obra de RDR". En F. Liernur, Arquitectura, en teoría. Escritos 1986-2010 (pp. 189-247). Buenos Aires, Argentina: Nobuko.
Malpas, J. (1999). Place and Experience: A Philosophical Topography. Cambridge, UK: Cambridge University Press.

Owen, C. (2014). "Forget everything you have learnd about art, and start from the beginnig": Charismatic leadership and art school teaching. Art, Design and Communication in Higher Education, 13 (2), pp. 201-214.

Uribe, J.L. (2011). "La escuela de Arquitectura del Universidad de Talca: un modelo de educación”. Dearq (9), pp. 62-73.

Vélez, S.R., Presman, I.J., Monje, R., Caldana, V., Valassina, S., Hernández, O.E., Ortega, L., Cordero, O., Ortiz, A.A., Spiridonidis, C., Valladares, C.E., Ortega, R., Baracco, J. \& Bausero, C. (2013). Educación superior en América Latina: reflexiones y perspectivas en Arquitectura. Recuperado de: http://tuningacademy. org/wp-content/uploads/2014/02/RefArchitecture LA_SP.pdf

Wit (de), H., Jaramillo, I.C., Gacel, J. \& Knight, J. (2005). Higher Education in Latin America. The International Dimension. Washington: The World Bank. Recuperado de: [http://siteresources.worldbank.org/ EXTLACREGTOPEDUCATION/Resources/Higher Ed_in_LAC_Intnal_Dimension.pdf]. 